\title{
Natural Products Isolation: A Challenge and Redressal
}

\author{
Anupam Maurya* and Subash C. Verma \\ ${ }^{1}$ Pharmacopoeia Commission for Indian Medicine \& Homoeopathy, Ministry of Ayush, India
}

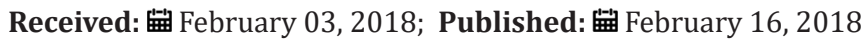

*Corresponding author: Anupam Maurya, Scientific Officer (Phytochemistry), Pharmacopoeia Commission for Indian Medicine \& Homoeopathy, Ministry of Ayush, Govt. of India, Ghaziabad-201002, India, Email: manupamk@gmail.com

\section{Importance}

Natural Products (NPs) are Chemical compounds directly found from the nature or can say from life. Chemical compounds are the secondary metabolites do not take parts any physiological function of plant and organism, the plant produces these secondary metabolites in stress for defence mechanism against herbivory and other inter-species defences. Various groups of NPs (Chemical compounds) are found in the plant and Animal, but the plant kingdom is very diverse and broad in presence of these chemical compounds (Phytomolecules). Last 20 years, almost 50\% drugs directly or indirectly derived from NPs for Human welfare, some of them are: Morphine, Penicilline G, Colchin, Pacitaxel, Atorvastatin, Vinblastine and Vincristine etc. NPs have important pharmacological activities and act as active component for both traditional and modern medicines and open a rout of drug discovery and development by providing the lead molecules.

\section{Challenges}

All the NPs exist as mixture with other compounds, often very complex mixture from which the product of interest must be isolated and purified. The isolation and purification of these molecules from the plants is very tedious and time taking for Natural Products Chemist. At present the used adsorbents $\mathrm{SiO}_{2}$ and $\mathrm{Al}_{2} \mathrm{O}_{3}$ are not chemical inert. Separation of natural products on alumina or silica gel sometimes results in recovery of only 70 $90 \%$. Sometimes severe losses of valuable material results because of irreversible adsorption on a solid support. In addition, isolation of artifacts has also been reported due to chemical reaction of the substrates with solid phase adsorbents. The Isolation \& purification of Phyomolecules (NPs) in sufficient quantities for structure elucidation derivatization, biological activities and other research purpose are paramount concern.

\section{Redressal}

Introduction of Counter Current Chromatography (CCC) provided new dimension in area of separation, isolation and purification of NPs. The Counter Current Chromatography is Partition type Chromatographic (liquid-liquid) techniques, in which both stationary and mobile phase are liquid. The separation of Compounds in CCC is based on their Partition Coefficient (K), in which the compounds distribute between biphasic solvent systems. CCC having number of advantages when it compared with the more traditional liquid-solid separations method:

(i) It can be used in both normal and reverse phase by simply switching the mobile into stationary phase.

(ii) It is less time taking

(iii) No irreversible adsorption due absence of solid stationary phase.

(iv) Recovery of injected sample is more than $90 \%$.

(v) Tailing minimized.

(vi) Low risk of sample denaturation.

(vii) Uniquely applicable to sensitive (unstable) compounds.

(viii) Low solvent consumption.

(ix) High loading capacity.

(x) Favorable economics (after initial investment, no expensive columns are required and only common solvents are consumed).

Table 1: Phytomolecules isolated by liquid-liquid chromatography with high purities.

\begin{tabular}{|c|c|c|}
\hline Natural Compounds & Techniques & Purity \\
\hline Ursolic acid & FCPC & $95.4 \%$ \\
\hline Piperine & HSCCC & $98.72 \%$ \\
\hline Squalene & FCPC & $95.5 \%$ \\
\hline Lysergol & FCPC & $97 \%$ \\
\hline Puerarin & FCPC & $99.0 \%$ \\
\hline$\gamma$-schisandrin & HSCCC & $96 \%$ \\
\hline gallic acid & HSCCC & $97 \%$ \\
\hline
\end{tabular}


With these advantages, CCC is gaining popularity as modern separation technique, especially in the bioassay-guided fractionation and separation of natural products as well as purification of analytical reagents and standard reference materials. It has been successfully used for the separation of a wide variety of natural products, alkaloids, terpenoids, flavonoids, steroidal glycoalkaloids, saponins, structural and geometrical isomers, semisynthetic and chiral compounds. Some important molecules isolated by liquidliquid chromatography (CCC) are in Table 1.

Table 2: Techniques and their manufacturers.

\begin{tabular}{|c|c|}
\hline Techniques & Manufactures \\
\hline FCPC & $\begin{array}{c}\text { Kromaton Technologies } \\
\text { http://www.kromaton.com }\end{array}$ \\
\hline HPCCC & $\begin{array}{c}\text { Dynamic Extractions (http://www.dynamicextrac- } \\
\text { tions.com }\end{array}$ \\
\hline $\begin{array}{c}\text { HSCCC, HPCCC, } \\
\text { CPC }\end{array}$ & AECS \\
\hline HSCCC & http://www.quattroprep.com/ \\
\hline Shanghai Tauto Biotech Co., Ltd. \\
\hline
\end{tabular}

We also successfully applied FCPC techniques for the separation and isolation of various phytomolecules likeclavine alkaloids. indole alkaloids, steroidal glycoalkaloids, triterpenoids, iridoids and nitrile glycosides as well enrichment many bioactive fractions. The largescale separations of these phytomolecules and enriched fractioned was used in chemical derivatization for study Structure Activity Relationship (SAR) and testing in-vivo and in-vitro activities. It is a rapid, simple and accurate technique having high scalability (mg to $\mathrm{kg}$ ), its popularity has grown worldwide. Due to high demand, liquid-liquid chromatographic techniques has evolved rapidly with advancement from initial Droplet counter-current chromatography (DCCC) and rotation locular counter-current chromatography (RLCCC) to new generations of instruments as high-speed CCC (or HSCCC) and high-performance CPC (or HPCPC). Some manufacturers of these techniques are summarized in Table 2 . The $80 \%$ of the world population relies on herbal medicine, asa result of increasing demands for herbal medicines; there are increasing concerns about the safety, standardization, efficacy and quality of these medicines. Due to Paucity of chemical markers (phytomolecules), the marker based standardization of these medicines is challenging task. The CCC technique may be played an important role to make availability of chemical markers from very complex mixture of NPs, which can be used for marker based standardization of herbal medicines.

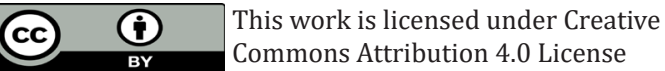

To Submit Your Article Click Here:

Submit Article

DOI: 10.32474/AOICS.2018.01.000117

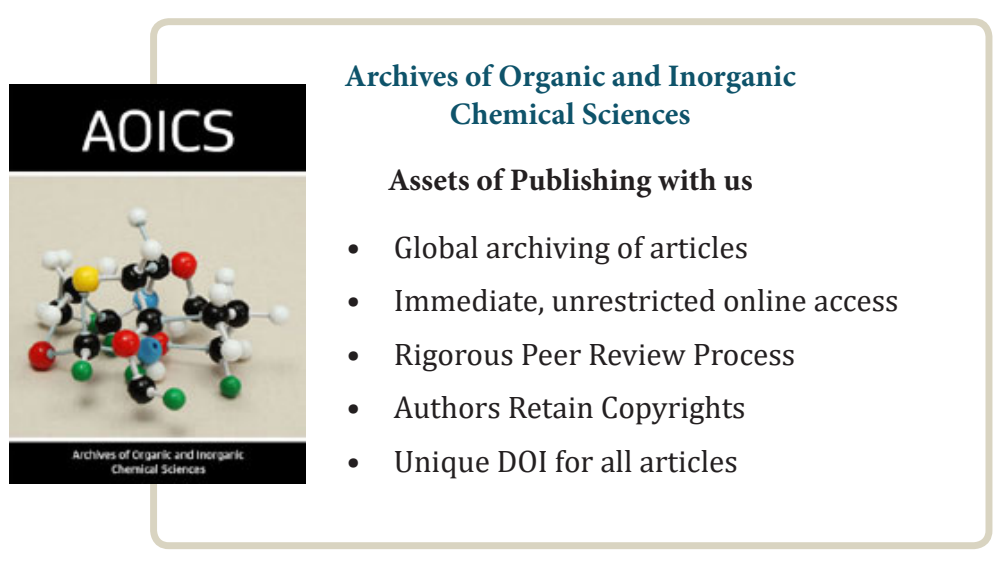

